\title{
STELLAR DYNAMICS OF NEEDLES
}

\author{
Scott Tremaine \\ Canadian Institute for Theoretical Astrophysics \\ University of Toronto, Toronto M5S 1A1, Canada \\ Tim de Zeeuw \\ The Institute for Advanced Study, Princeton, USA
}

\begin{abstract}
One dimensional "needles" are a limiting case of general triaxial stellar systems. Self-consistent, finite needles can have arbitrary longitudinal density distributions but have a fixed, universal distribution function. All needles are stable to all longitudinal perturbations but neutral to transverse perturbations.
\end{abstract}

\section{POTENTIAL THEORY}

Consider an axisymmetric stellar system with density $\rho(R, z)$, where $(R, \phi, z)$ are the usual cylindrical coordinates. We may always write the density in the form

$$
\rho(R, z)=\frac{\lambda(z)}{R_{0}^{2}(z)} F\left(\frac{R}{R_{0}(z)}, z\right)
$$

where $\lambda(z)=2 \pi \int R \rho(R, z) d R$ is the linear density, $R_{0}(z)$ is the boundary of the system, and the normalization condition is $2 \pi \int F(s, z) s d s=1$. We assume that the system is very prolate, so that the characteristic length of the needle is $Z \gg R_{0}$. Thus, there is a region in radius, $R_{0}<R \ll Z$, where the $z$-dependence of the potential $\Phi(R, z)$ resembles that of a wire of linear density $\lambda(z)$ :

$$
\Phi(R, z)=2 G \lambda(z) \ln R+\Phi_{0}(z) .
$$

Now shrink the system to a needle by setting $R_{0}(z)=\epsilon h(z) / Z$, where $\epsilon \rightarrow 0$. The difference between the potential on the axis and at the boundary of the needle is independent of $\epsilon$, and hence with a fractional error of order $C^{-1}$, with $C \equiv-\ln \epsilon$, we may write the potential anywhere inside the needle as

$$
\Phi(z)=-2 G C \lambda(z) .
$$

Note that this approximation fails in a small region near the tip where $\lambda=O\left(C^{-1}\right)$. 


\section{EQUILIBRIUM}

The distribution of stars in a needle-shaped stellar system is described by the distribution function $f(z, v)$, where $v$ is the velocity in the $z$-direction and $f(z, v) d z d v$ is the mass in the phase space interval $(z, v) \rightarrow(z+d z, v+d v)$. According to Jeans' theorem, the distribution function must have the form $f(z, v)=f(E)$ where $E=\frac{1}{2} v^{2}+\Phi(z)$ is the energy. A self-consistent needle satisfies

$$
\lambda(z)=\int_{-\infty}^{\infty} f(E) d v
$$

This integral equation for $f(E)$, together with equation (3) relating the density and potential, can be solved to yield

$$
f(E)= \begin{cases}\frac{1}{\pi G C}\left(-\frac{1}{2} E\right)^{1 / 2}, & E<0, \\ 0, & E>0 .\end{cases}
$$

Thus the distribution function for stellar needles is unique, i.e., all stellar needles have the same distribution function, independent of the density distribution $\lambda(z)$.

\section{STABILITY}

Antonov's (1961) stability criterion for spherical stellar systems with $f=f(E)$ has a straightforward analog for needles: the needle is stable to disturbances along the $z$-axis if the functional

$$
I[\eta]=\int \frac{[\eta(z, v)]^{2}}{|d f / d E|} d z d v+\int \eta(z, v) \Phi_{1}(z) d z d v \geq 0
$$

for all $\eta$, where $d f / d E<0$ and $\Phi_{1}(z)$ is the potential arising from a density $\lambda_{1}(z)=$ $\int \eta\left(z, v^{\prime}\right) d v^{\prime}$. For the needle we find $\left.I \mid \eta\right] \geq 0$, which proves stability to longitudinal disturbances. Of course, the needle is neutrally stable to disturbances which alter $\lambda(z)$ but leave the distribution function unaffected, since these simply transform the needle into a different equilibrium state.

The needle is neutrally stable to bending displacements. This result shows that the stability of very prolate systems to bending disturbances cannot be determined in the needle approximation.

\section{REFERENCE}

Antonov, V.A., 1961. Astr. Zh., 37, 918 (Translated in Sov. Astron., 4, 859). 